Supporting Information

\title{
High Yield Controlled Synthesis of Nano-Graphene Oxide by Water Electrolytic Oxidation of Glassy \\ Carbon for Metal-Free Catalysis
}

Qinwei Wei, ${ }^{\dagger,+}, / /$ Songfeng Pei, ${ }^{\dagger}, / /$ Guodong Wen, ${ }^{\dagger}$ Kun Huang, ${ }^{\dagger}$ Zhaohong Wu, ${ }^{\dagger,+}$ Zhibo Liu, ${ }^{\dagger}$ Wei Ma, ${ }^{\dagger,+}$ Hui-Ming Cheng, ${ }^{\dagger, t, \xi}$ Wencai Ren ${ }^{*}+,+$

†Shenyang National Laboratory for Materials Science, Institute of Metal Research, Chinese Academy of Sciences, Shenyang 110016, P. R. China.

\School of Materials Science and Engineering, University of Science and Technology of China, Shenyang 110016, P. R. China.

§Tsinghua-Berkeley Shenzhen Institute (TBSI), Tsinghua University, 1001 Xueyuan Road, Shenzhen 518055, P. R. China.

*Correspondence to: wcren@imr.ac.cn. 
a

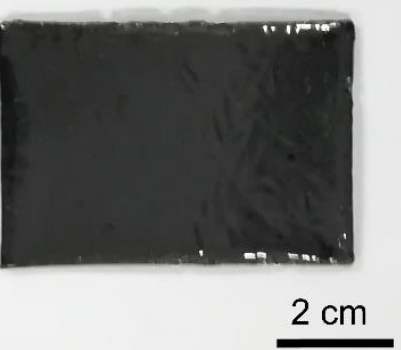

b

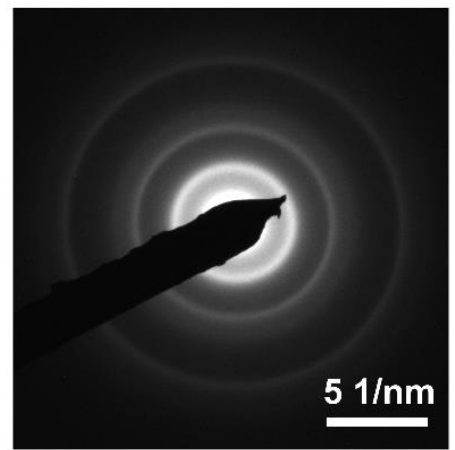

Figure S1. (a) Photo of a GC. (b) Electron diffraction pattern of GC, showing nanocrystalline structure with random orientations. 

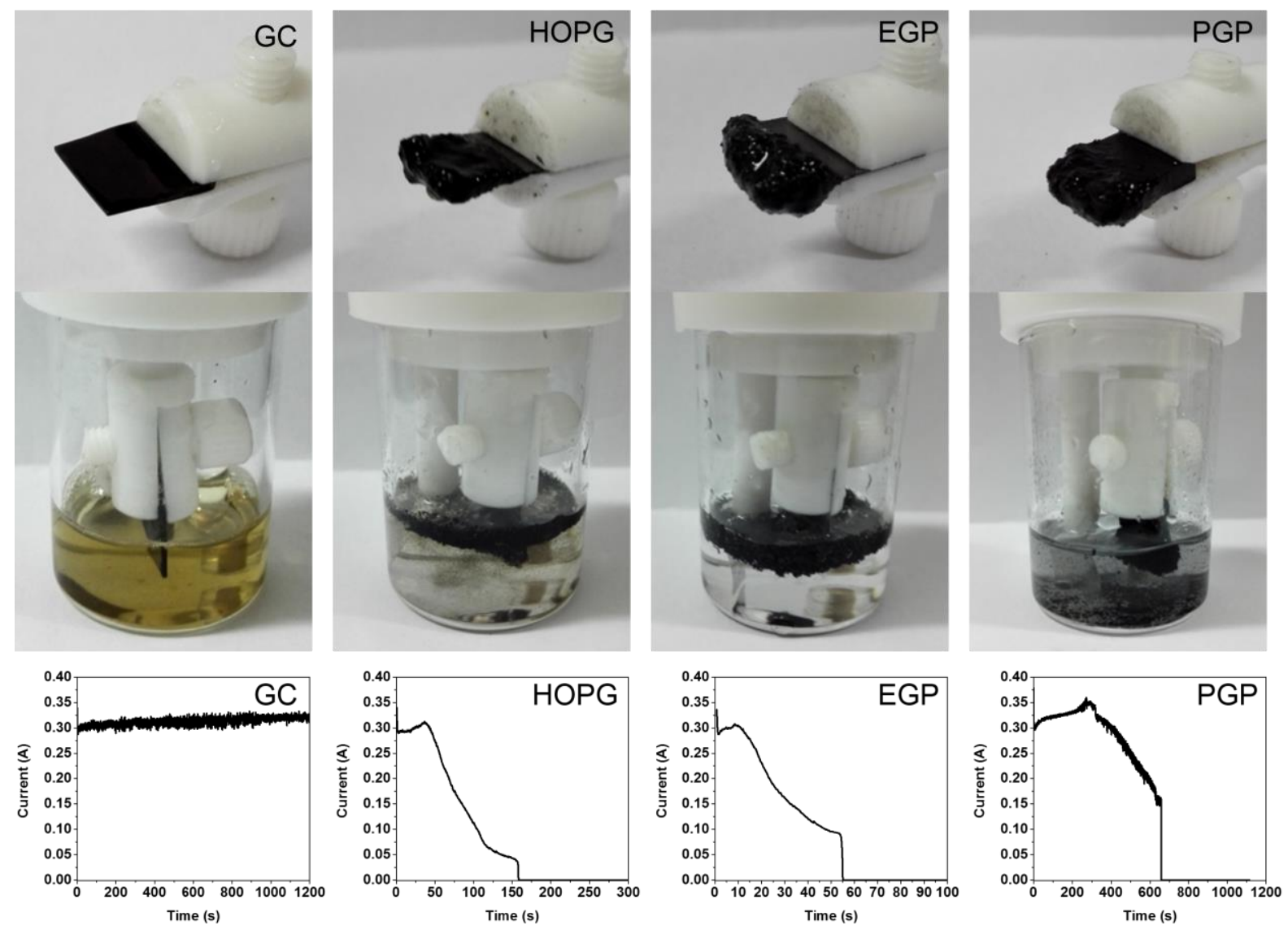

Figure S2. Photos of electrodes and electrolytes after EC reaction and the corresponding currenttime curves for different carbon materials as anodes in $50 \mathrm{wt} \% \mathrm{H}_{2} \mathrm{SO}_{4}$ solution with a constant potential of $3.5 \mathrm{~V}$. 
a

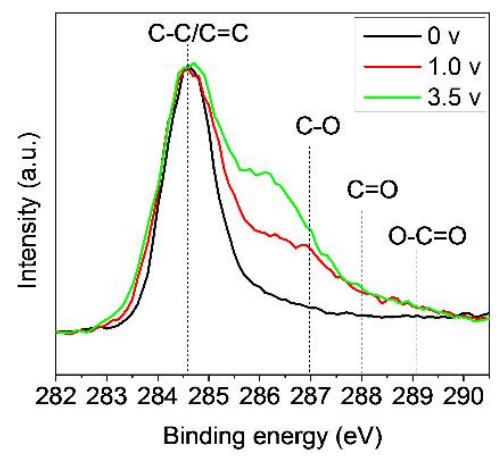

b

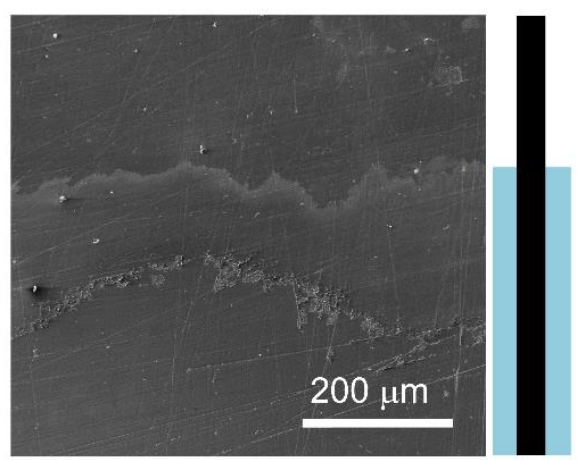

Figure S3. (a) High-resolution C1s XPS spectra of the GC surface after EC reaction with a potential of $0 \mathrm{~V}$ (black line), $1.0 \mathrm{~V}$ (red line) and $3.5 \mathrm{~V}$ (green line). (b) SEM image of GC surface at the junction area above and below the electrolyte after EC reaction for $30 \mathrm{~min}$, showing that the exfoliation of GC anode proceeds from the surface. 

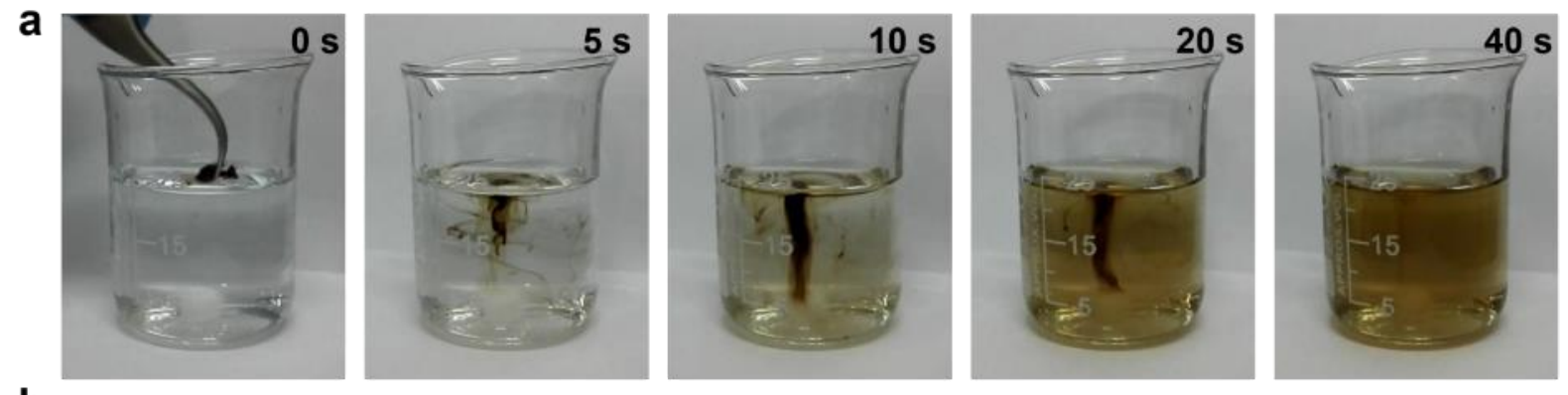

b
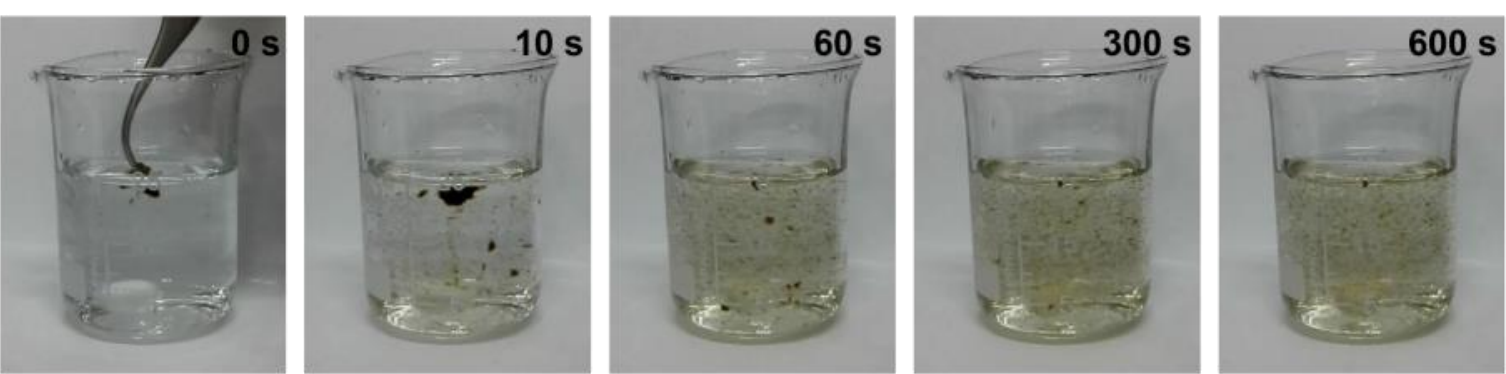

C
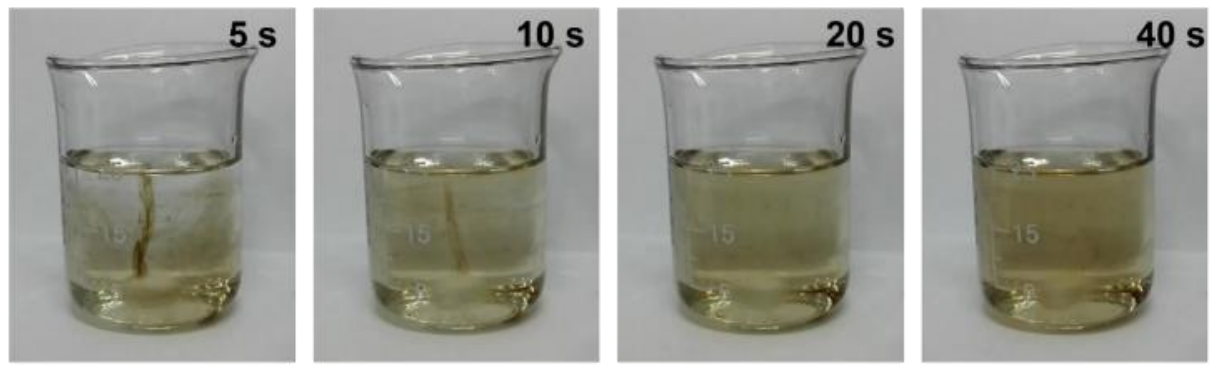

d
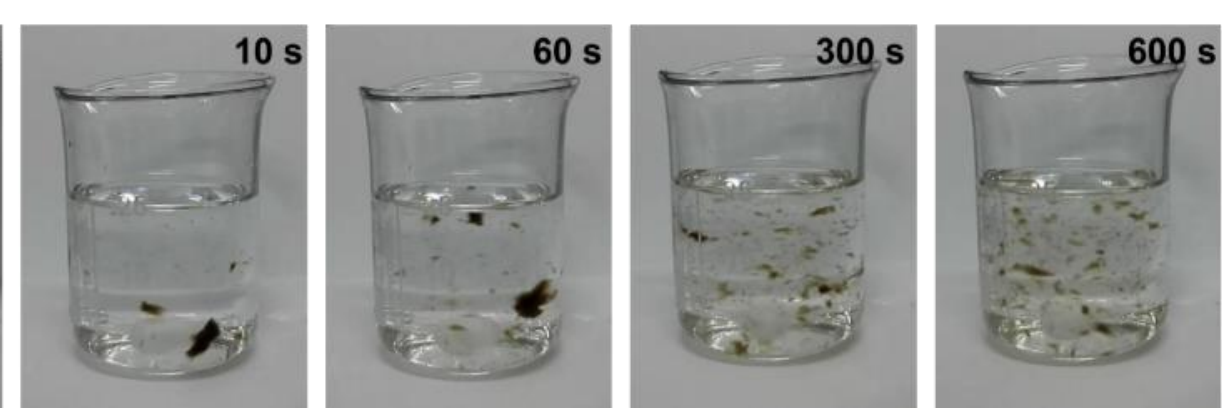

Figure S4. The photos showing the dispersion of ENGO in water (a), HGO in water (b), ENGO in ethanol (c) and HGO in ethanol (d). Note that the ENGO powders can be completely dispersed in water and ethanol within $40 \mathrm{~s}$ by stirring. However, the HGO cannot be well dispersed in water and ethanol even after $600 \mathrm{~s}$ without sonication. 
a

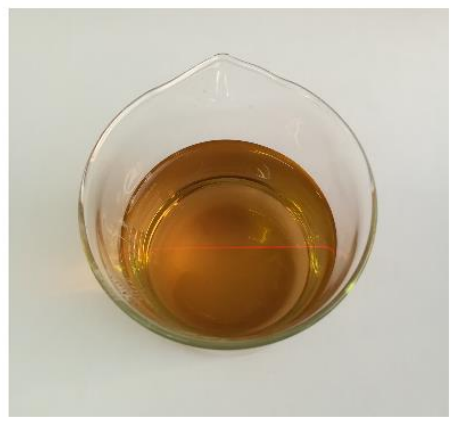

C

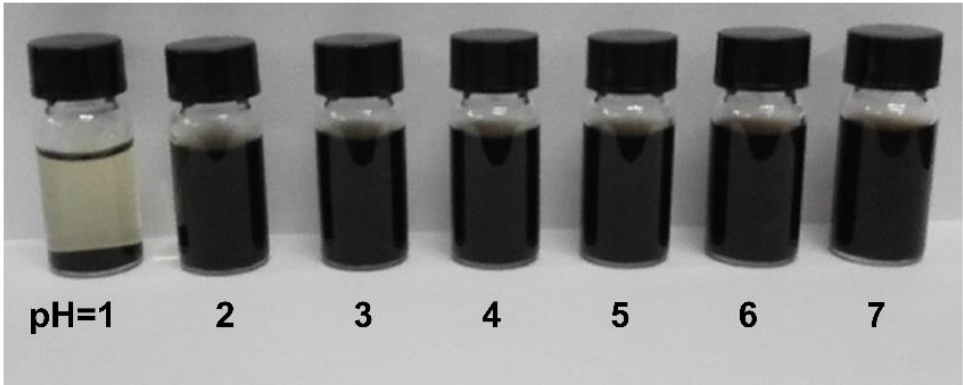

Figure S5. (a) The Tyndall effect of ENGO aqueous solution. (b) The Zeta potential of ENGO and HGO solution as a function of $\mathrm{pH}$ value. (c) Photo showing the dispersion state of ENGO aqueous solution with various $\mathrm{pH}$ values after storage for two months. 

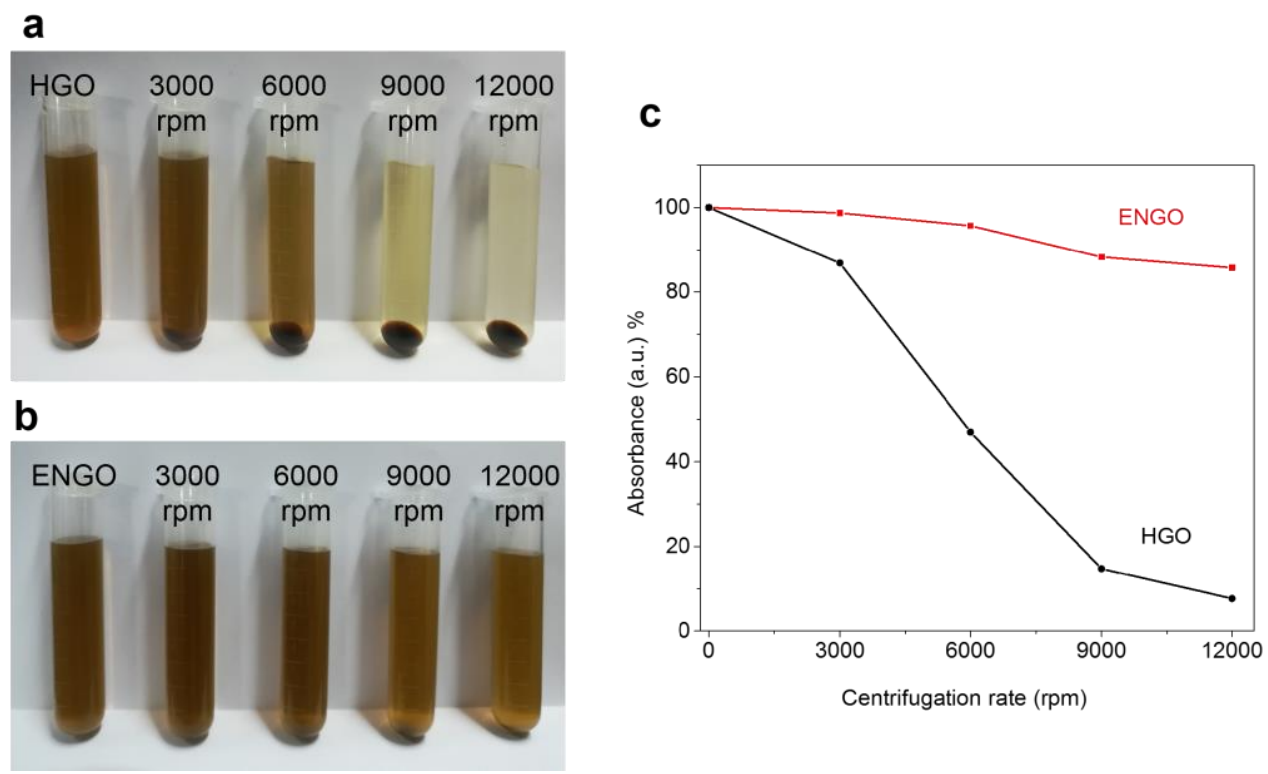

Figure S6. Stability of ENGO and HGO solution at a pH value of 3. $(a, b)$ The photos of ENGO (a) and HGO (b) solution after centrifugation respectively at 0, 3000, 6000, 9000 and $12000 \mathrm{rpm}$ for $30 \mathrm{~min}$. (c) The UV-vis absorbance of ENGO and HGO suspension at $230 \mathrm{~nm}$ after centrifugation at different rates. 
a

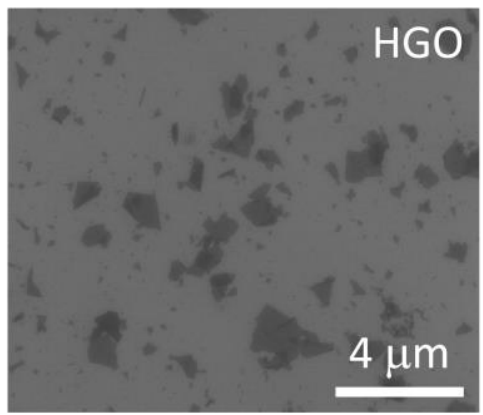

b

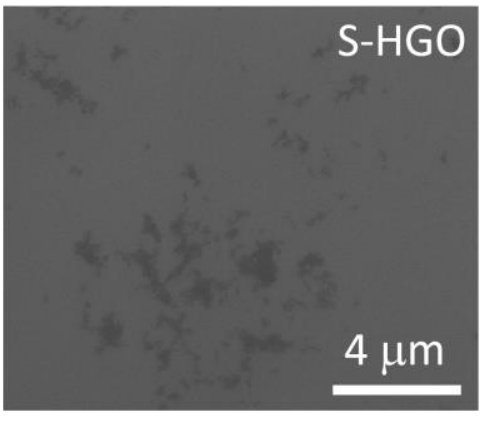

C

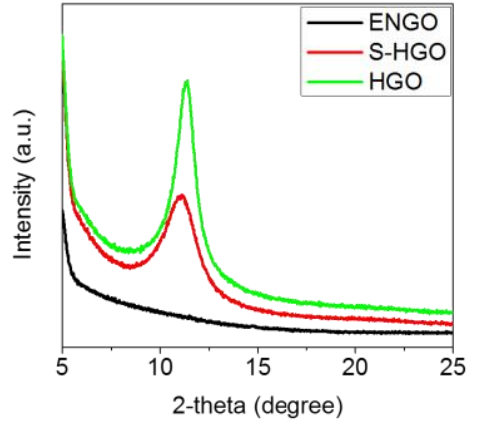

Figure S7. SEM images of HGO (a) and S-HGO (b). (c) Comparison of XRD patterns of ENGO, HGO and S-HGO powders. The small size HGO (S-HGO) was synthesized by ultrasonication of HGO solution for five hours. The pristine HGO and S-HGO have a mean size of $\sim 2 \mu \mathrm{m}$ and $\sim 0.5$ $\mu \mathrm{m}$, respectively. Note that both HGO and S-HGO powders show a characteristic XRD peak at $\sim 11.3^{\circ}$, corresponding to an interlayer spacing of $\sim 0.78 \mathrm{~nm}$. The layered structure in HGO/SHGO powders is formed by the restacking of HGO/S-HGO sheets during drying. Restacking is a common characteristic of 2D materials because of their high specific ratio (size/thickness). Both the intensity and width of XRD peak are related to the order degree of restacked structure. Bigger GO sheets can form restacked structure with better ordering, leading to strong and sharp XRD peak, while smaller GO sheets result in weaker and broader XRD peak. Different from HGO and S-HGO powders, ENGO powder shows no characteristic XRD peak, indicating no restacking for very small ENGO. 
a
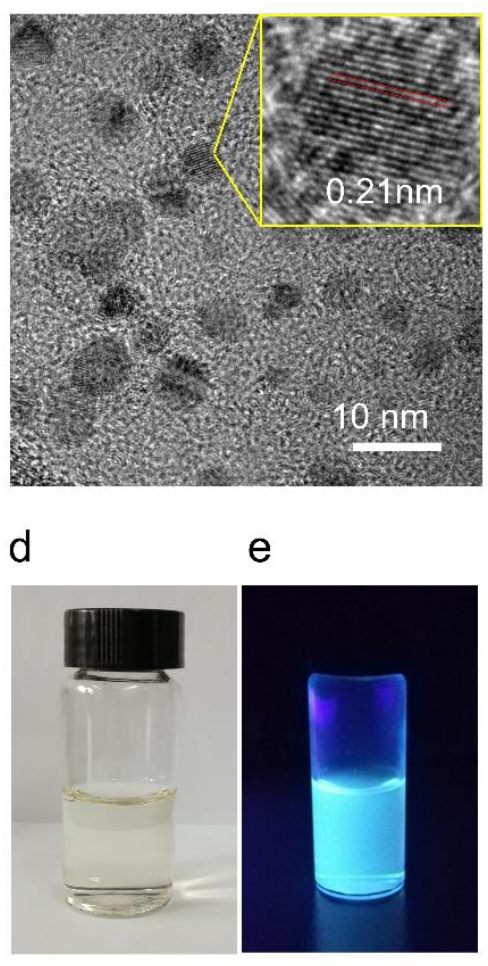

b

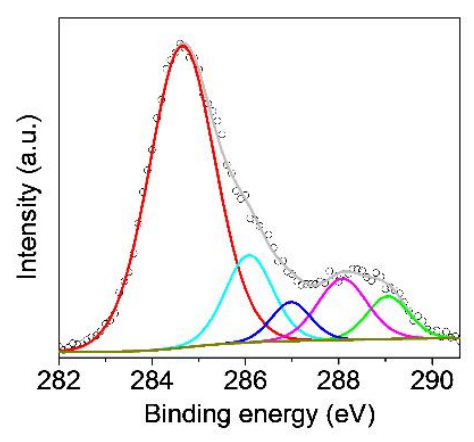

$f$

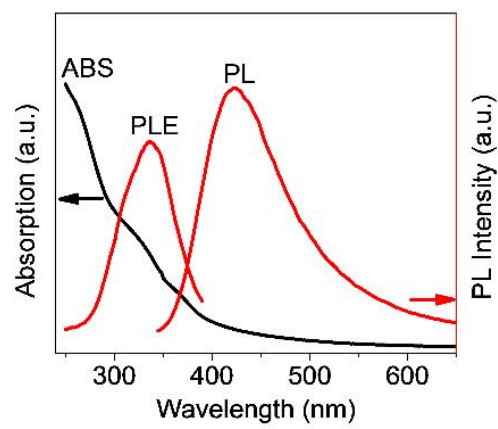

C

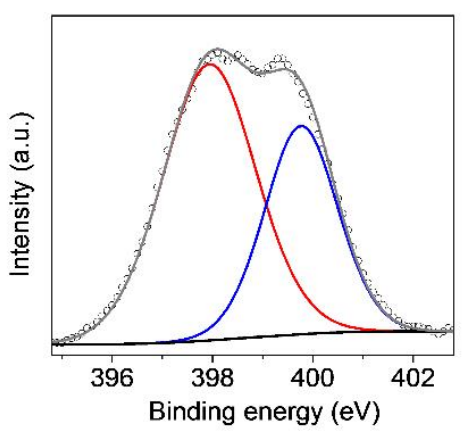

g

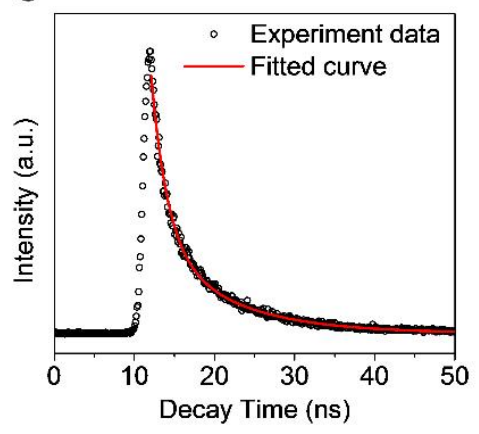

Figure S8. Characterizations of the reduced ENGO (RENGO) by hydrothermal treatment in ammonia solution (5.6 wt\%). (a) TEM image of RENGO, clearly showing the lattice fringers of graphite with a d spacing of $0.21 \mathrm{~nm}$. (b, c) High-resolution C1s (b) and N1s (c) XPS spectra of RENGO. The greatly reduced $\mathrm{C} 1$ s peaks related to carbon-oxygen bonding confirm the reduction of ENGO. The presence of both pyridine-like $(398.0 \mathrm{eV})$ and pyrrolic-like $(399.7 \mathrm{eV})$ nitrogen atoms indicates that ENGO is doped by nitrogen. (d, e) Photos of RENGO aqueous solution under visible light (d) and UV light with $365 \mathrm{~nm}$ excitation (e), showing a strong blue photoluminesence (PL). (f) UV-vis spectra, PL emission spectra under an excitation wavelength at $330 \mathrm{~nm}$ and PL excitation (PLE) spectra under an emission wavelength at $425 \mathrm{~nm}$ for RENGO aqueous solution. (g) Time-resolved PL spectra. The lifetime data of RENGO can be well fitted to a double exponential function with $\tau_{1}=2 \mathrm{~ns}$ and $\tau_{2}=9 \mathrm{~ns}$. 


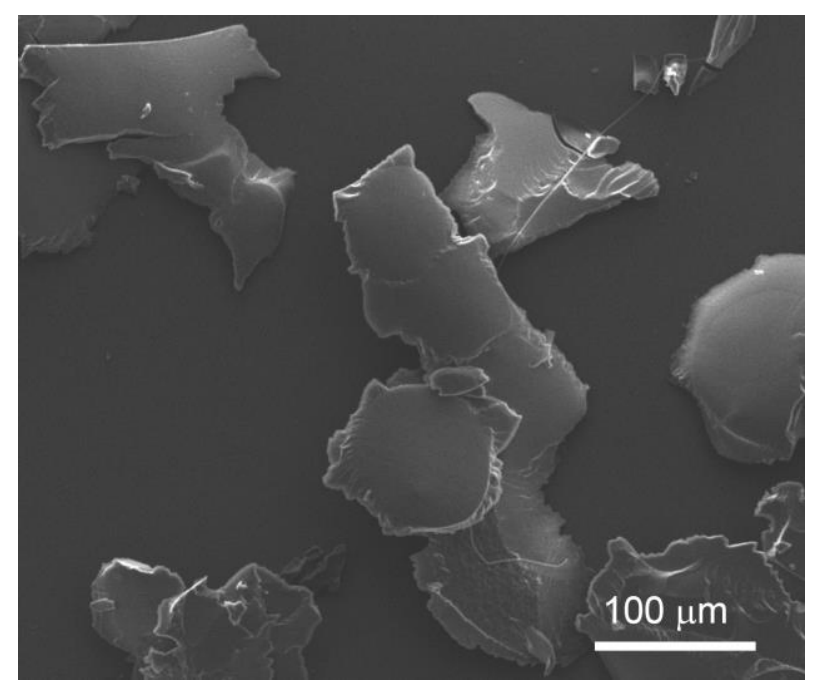

Figure S9. SEM image of black particles obtained after EC reaction in $10 \mathrm{wt} \%$ sulfuric acid solution, showing a typical fracture character of brittle materials. 

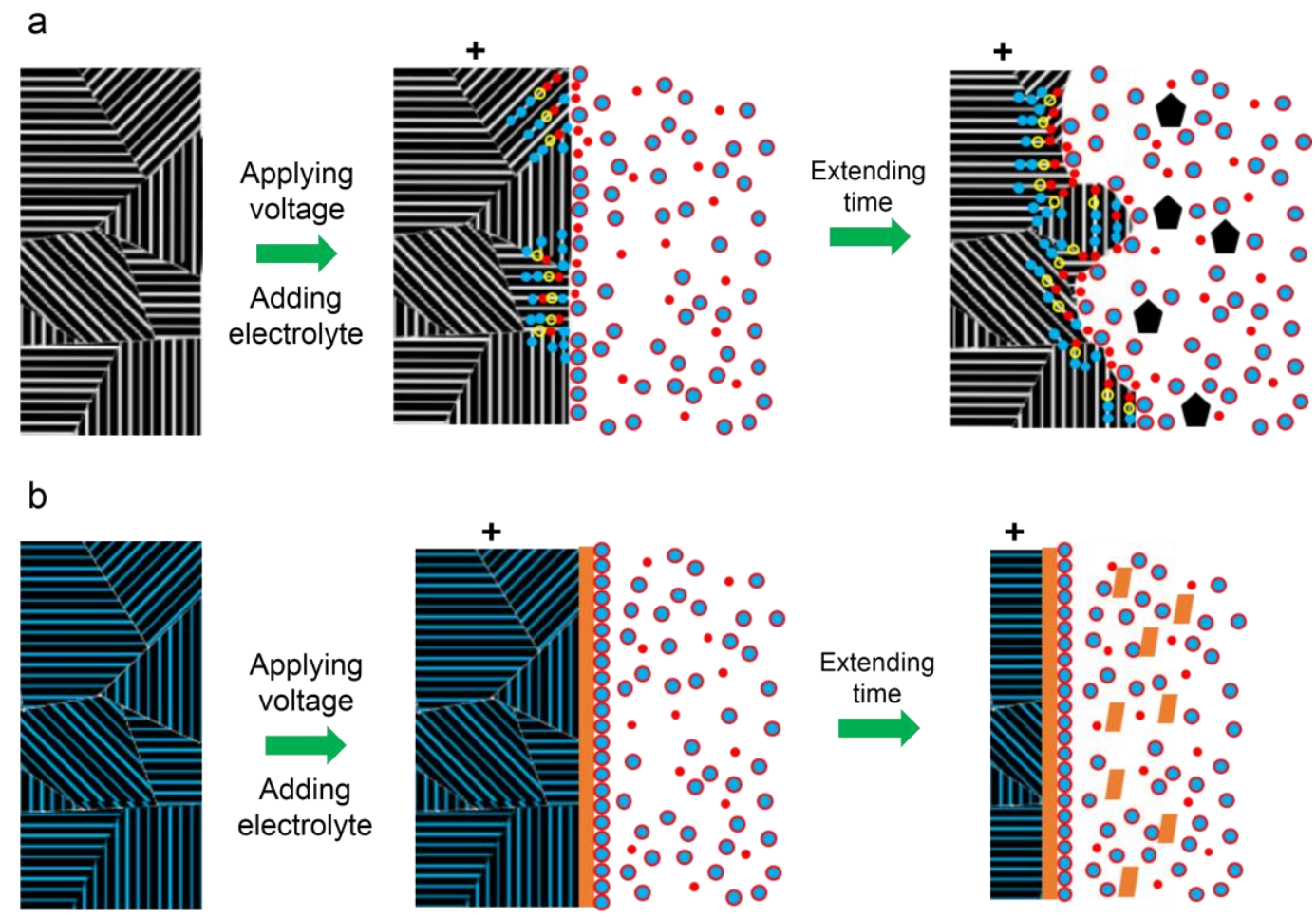

\begin{tabular}{|lccc|}
\hline EGP & GICP & o Hydrate $\mathrm{H}_{2} \mathrm{SO}_{4} / \mathrm{SO}_{4}{ }^{2-} / \mathrm{HSO}_{4}^{-}$ & $\bullet \mathrm{H}_{2} \mathrm{O}$ \\
$\mathrm{Gas}$ & $\bullet \mathrm{H}_{2} \mathrm{SO}_{4} / \mathrm{SO}_{4}{ }^{2-} / \mathrm{HSO}_{4}^{-}$ & - Graphite/graphene & $\| \mathrm{GO}$ \\
\hline
\end{tabular}

Figure S10. The schematic of the oxidation and exfoliation mechanism of EGP (a) and stage-I $\mathrm{H}_{2} \mathrm{SO}_{4}$ graphite intercalation compound paper (b, GICP) in $50 \mathrm{wt} \%$ sulfuric acid electrolytes. 


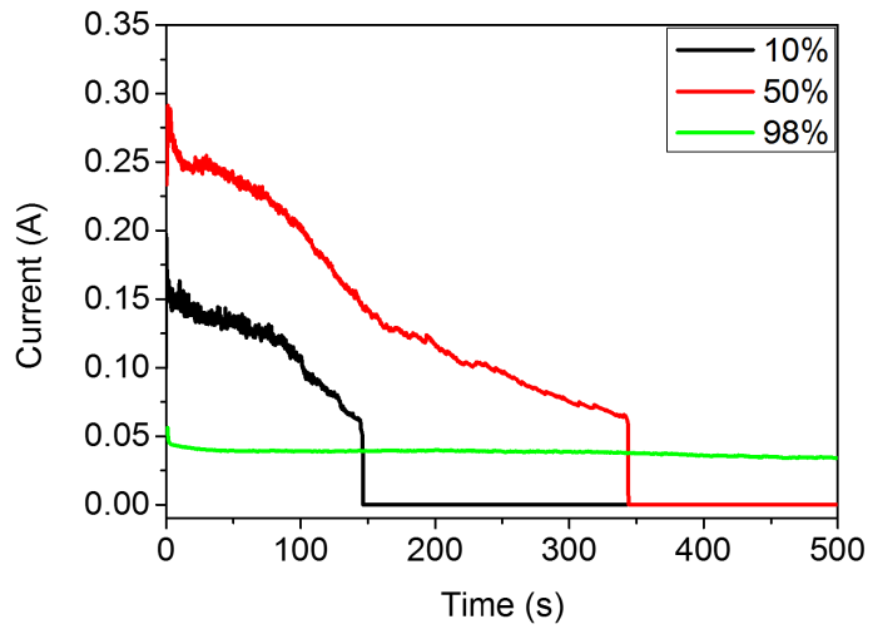

Figure S11. Current change as a function of reaction time for GICP in the electrolytes with different $\mathrm{H}_{2} \mathrm{SO}_{4}$ concentrations (10 wt $\%, 50 \mathrm{wt} \%$, and $98 \mathrm{wt} \%$ ) at a constant potential of $3.5 \mathrm{~V}$. 

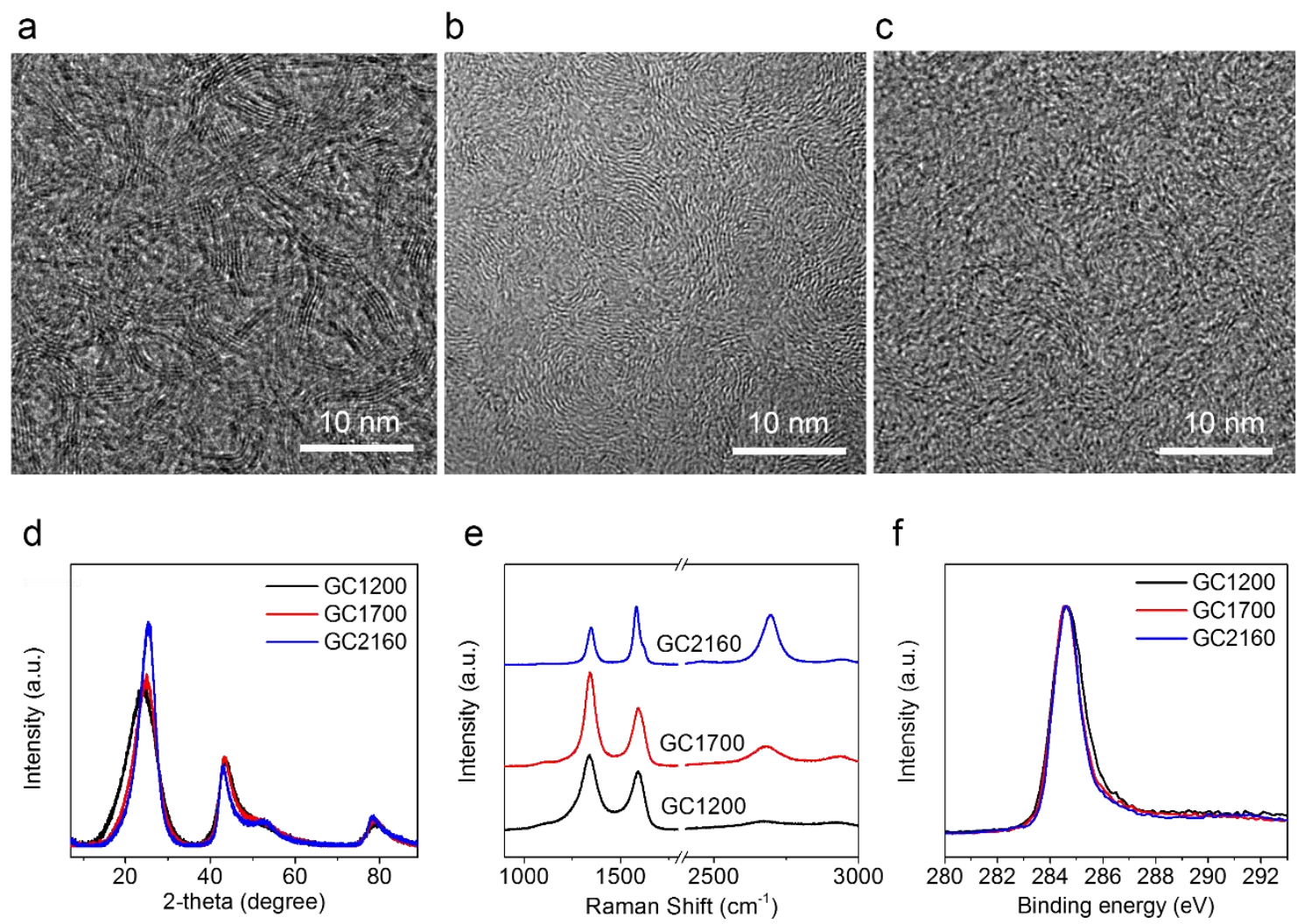

Figure S12. Characterization of GC synthesized with different graphitization temperatures. (a-c)

TEM images of GC synthesized with graphitization temperature of $2160{ }^{\circ} \mathrm{C}$ (a, GC2160), 1700

${ }^{\circ} \mathrm{C}$ (b, GC1700) and $1200{ }^{\circ} \mathrm{C}$ (c, GC1200). (d-f) XRD patterns (d), Raman spectra (e), and $\mathrm{C} 1 \mathrm{~s}$ XPS spectra (f) of GC2160, GC1700, and GC1200. 
Table S1. The peak fitting results of C1s XPS spectra of ENGO2160, ENGO1700 and ENGO1200.

\begin{tabular}{lllll}
\hline Samples & $\mathrm{C}=\mathrm{C} / \mathrm{C}-\mathrm{C}$ & $\mathrm{C}-\mathrm{O}$ & $\mathrm{C}=\mathrm{O}$ & $\mathrm{O}-\mathrm{C}=\mathrm{O}$ \\
& $($ Area $\%)$ & $($ Area $\%)$ & $($ Area $\%)$ & $($ Area $\%)$ \\
\hline ENGO2160 & 55.4 & 30.53 & 6.79 & 7.28 \\
ENGO1700 & 56.63 & 28.21 & 7.33 & 7.82 \\
ENGO1200 & 58.76 & 25.71 & 7.51 & 8.03 \\
\hline
\end{tabular}

